\title{
Titel: LANDESSTRAFVOLLZUGSGESETZE
}

\author{
Von Heinz Cornel
}

\section{Die Entwicklung des deutschen Strafvollzugs nach der Föderalismusreform des Jahres 2006 mit der Verlagerung der Gesetzgebungskompetenz auf die Bundesländer}

\section{Anlesetext NK 1/2018}

Zehn Jahre nach der Föderalismusreform mit der Verlagerung der Gesetzgebungskompetenz für den Justizvollzug vom Bund auf die Länder ist es Zeit, Bilanz zu ziehen: Was haben mehr als 60 neue Justizvollzugsgesetze mit ganz verschiedenem Zuschnitt (Einzelgesetze für Untersuchungshaft, Jugendstrafvollzug, Strafvollzug und Sicherungsverwahrung oder Landesjustizvollzugsgesetze, die alle Bereiche umfassen) gebracht?

Bekanntlich war diese Verlagerung der Gesetzgebungskompetenz ohne vorherige inhaltliche fachliche Debatte als ein kleiner Teil eines Kompromisses im Zuge der Föderalismusreform zustande gekommen. Angesichts der Tatsache, dass in jener Zeit in der Landespolitik ohnehin schon populistische Forderungen nach einem härteren Strafvollzug Konjunktur hatten, wurde daran schnell Kritik geübt.

Aus dem Rechtsausschuss des Deutschen Bundestages sprachen sich verschiedene Mitglieder, wie beispielsweise Siegfried Kauder von der CDU, Sabine LeutheusserSchnarrenberger von der FDP, Hans Christian Ströbele von den Grünen, Wolfgang Neskovic von der PDS, Richter am Bundesgerichtshof a.D. und stellvertretender Vorsitzender des Rechtsausschusses gegen die drohende Verlagerung der Gesetzgebungskompetenz vom Bund auf die Länder aus. In mehreren Landesparlamenten und Bezirksparteitagen kritisierten SPD-Mitglieder „die Kleinstaaterei im Strafvollzug“. Auch der Richterbund, die Deutsche Vereinigung für Jugendgerichte und Jugendgerichtshilfen, der DBH-Fachverband für soziale Arbeit, Strafrecht und Kriminalpolitik, Verdi, die Landesarbeitsgemeinschaft der Psychologinnen und Psychologen im niedersächsischen Justizvollzug, die Arbeitsgemeinschaft der Leiter der Jugendstrafanstalten und der besonderen Vollstreckungsleiter, die Bundesarbeitsgemeinschaft der Sozialarbeiterinnen und Sozialarbeiter im Vollzug, die Frühjahrsvollversammlung der katholischen Bischöfe 2006 und die Bundesarbeitsgemeinschaft Straffälligenhilfe e.V. sprachen sich für die Beibehaltung der Gesetzgebungskompetenz für den Strafvollzug beim Bund 
aus. In einem offenen Brief forderten zwölf ehemalige Bundes- und Landesjustizminister aus ganz unterschiedlichen Parteien, unter anderem Lore Maria Peschel-Gutzeit, Sabine Leutheusser-Schnarrenberger, Herta Däubler-Gmelin, Hans Otto Bräutigam, Christian Pfeiffer, Eberhard Diepgen, Wolfgang Wieland, Rupert von Plottnitz und Klaus Klingner die Beibehaltung der Bundeskompetenz. Eine von mir damals formulierte Resolution mit der Überschrift „Gesetzgebungskompetenz für den Strafvollzug muss beim Bund bleiben“ wurde zur Jahreswende 2004/2005 von mehr als 120 Strafrechtswissenschaftlern, Strafvollzugsrechtlern und Kriminologen unterzeichnet. Nicht nur Frieder Dünkel und Horst Schüler-Springorum befürchteten einen ,Wettbewerb der Schäbigkeit', der hinsichtlich der Praxis des Strafvollzugs auf Länderebene längst im Gange war. In Hamburg ließ die Koalition von CDU mit der rechtspopulistischen Schill-Partei unter dem Justizsenator Roger Kusch die sozialtherapeutische Anstalt und die Übergangsanstalt Moritz-Liepmann Haus schließen und sorgte ab Oktober 2001 für einen härteren Strafvollzug mit weniger Öffnungen und Strafrestaussetzungen zur Bewährung. In Hessen hatte Ministerpräsident Roland Koch bereits 1999 im Wahlkampf für den härtesten Strafvollzug in Deutschland plädiert und sein Justizminister Christian Wagner bestätigte 2003, dass man das erreicht habe. Die Anzahl der Hafturlaube ging um zwei Drittel zurück und die der Strafrestaussetzungen zur Bewährung um ein Drittel.

Nach der Verlagerung der Gesetzgebungskompetenz wurden zunächst in Bayern (Dezember 2007), Hamburg (erste Fassung Dezember 2007), Niedersachsen (Dezember 2007), Baden-Württemberg (November 2009) und Hessen (Juni 2010) Strafvollzugsgesetze erlassen. Schon vorher gab es - ausgelöst unter anderem von der Rechtsprechung des Bundesverfassungsgerichts zum Jugendstrafvollzug vom 31.5.2006 (BVerfGE 116, S. 69ff.) - die ersten Gesetze zum Jugendstrafvollzug (März 2007 bis Dezember 2007) und Untersuchungshaftvollzug (Juli 2009 bis Dezember 2011). Wenige Wochen nach der Verabschiedung des Hessischen Jugendstrafvollzugsgesetzes im November 2007, das in der Fachliteratur durchaus positiv gewürdigt wurde, forderte der hessische Ministerpräsident für straffällig gewordene Kinder und Jugendliche die Inhaftierung in Erziehungslagern und hatte sich so die Poleposition im oben genannten Wettbewerb gesichert. Im August 2012 legten dann zehn der elf Länder, in denen das Bundesstrafvollzugsgesetz zu dieser Zeit noch galt (NRW war nicht beteiligt) einen Musterentwurf eines Landesstrafvollzugsgesetzes vor, der versprach, dem Wettbewerb der Schäbigkeit zu trotzen.

In $\ 2$ des Musterentwurfs wurde die Befähigung der Gefangenen, künftig in sozialer Verantwortung ein Leben ohne Straftaten zu führen, zum alleinigen Ziel definiert und der Angleichungsgrundsatz wurde sogar gegenüber dem Bundestrafvollzugsgesetz konsequenter formuliert. $\ 3$ Abs. 5 formulierte als zusätzlichen Grundsatz die Wahrung und Förderung des Bezugs der Gefangenen zum gesellschaftlichen Leben. Positiv war zu würdigen, dass bei Gefangenen, die eine Ersatzfreiheitsstrafe verbüßen, Möglichkeiten der Abwendung der Vollstreckung durch freie Arbeit oder ratenweise Tilgung der Geldstrafe zu erörtern und zu fördern sind, um auf eine möglichst baldige Entlassung hinzuwirken. Kritisiert wurden fehlende Regelungen zum Frauenvollzug, 
fehlende verbindliche Festlegungen benötigter Ressourcen, um gute Konzepte finanziell, organisatorisch, personell und institutionell abzusichern, sowie die erneute Zögerlichkeit, die Entlohnung deutlich anzuheben und Gefangene in die Sozialversicherungssysteme einzubeziehen, wobei hier die Zuständigkeit des Bundesgesetzgebers durchaus zu sehen ist. Kritisch ist auch zu sehen, dass der Musterentwurf in $\$ 78$ Abs. 2 Ziffer 4 den Entzug oder die Beschränkung des Aufenthalts im Freien als besondere Sicherungsmaßnahme ohne zeitliche Begrenzung entgegen Nr. 27.1 der European Prison Rules für zulässig erklärte (vgl. dazu auch den folgenden Beitrag von Manuel Mika).

Der Musterentwurf wurde in den zehn Bundesländern unterschiedlich umgesetzt. Neben eher formalen Variationen zum Datenschutz und zu den Genderformulierungen, die vor allem zu einer unterschiedlichen Nummerierung der einzelnen Paragraphen in den Gesetzen führten, gab es Differenzen in den Mindestbesuchszeiten, in den Voraussetzungen für Langzeitausgänge und dem Schusswaffengebrauch. Inhaltlich breiter diskutiert wurde die Abschaffung der Arbeitspflicht für Gefangene im Musterentwurf. Einige Bundesländer konnten sich dazu nicht durchringen - mit ganz unterschiedlichen Argumenten, die hier nicht ausgebreitet werden können (vgl. dazu den folgenden Beitrag von Laura Isabelle Marisken).

Von den Arbeiten am Musterentwurf eines Landesstrafvollzugsgesetzes und dessen Umsetzung in den Ländern ging eine dreifache Botschaft aus:

- Mit bewussten kriminalpolitischen Entscheidungen kann man sich dem Wettbewerb der Schäbigkeit widersetzen. Die Bundesländer müssen nicht in einen Wettbewerb treten und fachliche Argumentationen können - bei aller Kritik im Einzelnen und Respektierung der Unterschiedlichkeit in einem breiten Spektrum - sich dem Populismus widersetzen.

- Der Verlagerung der Gesetzgebungskompetenz auf die Bundesländer folgte wohl in vielen Justizministerien die Einsicht, dass diese fachliche Kompetenz für eine eigenständige Gesetzeserstellung unter Würdigung der Erfahrungen aus dem deutschen und internationalen Strafvollzug sowie der aktuellen kriminologischen Diskurse kaum ausreicht. Die Bündelung der Kompetenzen war deshalb eine kluge Entscheidung, die freilich die Intention der Föderalismusreform diesbezüglich konterkariert.

- Neben den oben genannten Kritikpunkten am Musterentwurf selbst geht von den kleinen, in den Landesparlamenten beschlossenen Änderungen jeweils eine Botschaft aus. Wenn die Regelung des $₫ 38$ Abs. 3 Satz 2 des Musterentwurfs über die Voraussetzungen eines Langzeitausgangs für Gefangene, die zur lebenslangen Freiheitsstrafe verurteilt wurden, beispielsweise in Berlin, Rheinland-Pfalz, Saarland, Sachsen und Mecklenburg-Vorpommern derart geändert wurde, dass diese Langzeitausgang nicht nach mindestens fünf Jahren, sondern erst nach zehn Jahren erhalten können, so ist darin nicht nur ein Misstrauen gegenüber den Anstaltsleitungen und AutorInnen des Musterentwurfs zu sehen, sondern angesichts der geringen Relevanz auch ein Stück symbolische Politik. Das Verbot des Schusswaffengebrauchs durch Bedienstete innerhalb der Anstalt, das im Musterentwurf geregelt war, wurde 
in Brandenburg, Mecklenburg-Vorpommern, Rheinland-Pfalz und Sachsen übernommen, in Berlin jedoch entgegen fachlichem Rat der eigenen Verwaltung nicht eingeführt.

Schon im Vorfeld der Föderalismusreform hatten einzelne Bundesländer immer wieder versucht, als Ziel des Strafvollzugs den Schutz der Allgemeinheit der Befähigung, in sozialer Verantwortung ein Leben ohne Straftaten zu führen, voranzustellen. Bayern hat das in Art. 2 unter der Überschrift ,Aufgaben des Vollzugs' direkt nach der Föderalismusreform im eigenen Strafvollzugsgesetz umgesetzt. Hessen hat es bei der Benennung des Eingliederungsauftrags an erster Stelle belassen, aber die sichere Unterbringung und Beaufsichtigung während des Vollzugs gleichberechtigt hinzu gesetzt. Hamburg stellt explizit fest, dass die Befähigung zum Leben ohne Straftaten und der Schutz der Allgemeinheit gleichwertig sind und dass darin kein Gegensatz besteht. Sachsen regelt in $\$ 2$ seines Vollzugsgesetzes unter der Überschrift, Ziel und Aufgabe des Vollzugs' die Befähigung der Gefangenen zum Leben ohne Straftaten als Ziel und den Schutz der Allgemeinheit als Aufgabe und fügt dann als Satz 3 hinzu, dass dies durch eine zielgerichtete und wirkungsorientierte Vollzugsgestaltung sowie sichere Unterbringung und Beaufsichtigung der Gefangenen gewährleistet werde. Die anderen Länder folgen dem Bundesstrafvollzugsgesetz und Musterentwurf - sie nennen als Ziel die Befähigung, künftig in sozialer Verantwortung ein Leben ohne Straftaten zu führen und als Aufgabe, die Allgemeinheit vor weiteren Straftaten schützen.

Alle unterschiedlichen Formulierungen können nicht hinter die Position des Bundesverfassungsgerichts zurück, das die Resozialisierung als Verfassungsprinzip direkt aus der Würde des Menschen und dem Sozialstaatsprinzip abgeleitet hat. Das setzt dem Wettbewerb Schranken. Wie die Entwicklungen der letzten zehn Jahre diesbezüglich im Einzelnen erfolgten, soll in den folgenden vier Beiträgen zu den vollzugsöffnenden Maßnahmen, zur Gefangenenarbeit, zu Sicherungs- und Disziplinarmaßnahmen und dem Jugendarrest in einer Bilanz von zehn Jahren Föderalismusreform dargestellt werden:

Frieder Dünkel, Ineke Pruin, Paul Beresnatzki und Judith Treig beschäftigen sich mit den Veränderungen der vollzugsöffnenden Maßnahmen. Dies ist von ganz besonderer Relevanz, weil in den letzten 10-15 Jahren die Übergänge vor allem aus dem Strafvollzug in den Fokus der Kriminologie, der Kriminalpolitik und Straffälligenhilfe geraten sind. Sie betrachten Vollzugslockerungen als evidenzbasierte Strategie und im Lichte internationaler Standards, referieren die Regelungen im Erwachsenen- und Jugendstrafvollzug und stellen die tatsächliche Entwicklung des offenen Vollzugs und Hafturlaubs seit dem Inkrafttreten des Bundesstrafvollzugsgesetzes dar.

Laura Isabelle Marisken untersucht vollzugsrechtliche, verfassungs- und menschenrechtliche Aspekte der Arbeit und Arbeitsentlohnung in den Länderstrafvollzugsgesetzen. Dabei geht es um unterschiedliche Bewertungen des Resozialisierungsziels Arbeit, die Arbeitspflicht und die Arbeitsentlohnung.

Manuel Mika hat sich mit neueren Entwicklungen der besonderen Sicherungs- sowie der Disziplinarmaßnahmen im Strafvollzug beschäftigt. Er vergleicht die neuen 
Landesregelungen untereinander und mit dem alten Bundesstrafvollzugsgesetz, setzt sie in Bezug zu den European Prison Rules und scheut sich nicht, beispielsweise hinsichtlich des Arrestes die Verfassungsmäßigkeit anzuzweifeln.

Schließlich beleuchtet Anne Kaplan die neuere Entwicklung im Hinblick auf die Jugendarrestvollzugsgesetze. Sie erörtert die Entstehung des Jugendarrestvollzugs, gibt einen Überblick über die Gesetzgebungsverfahren in den einzelnen Bundesländern, bewertet die Gesetze anhand unterschiedlicher Kriterien und äußert sich in ihrem $\mathrm{Fa}$ zit dazu, inwieweit die Föderalismusreform hinsichtlich der Jugendarrestvollzugsgesetze einen Wettbewerb der Schäbigkeit ausgelöst hat.

Mit diesen vier Beiträgen werden wichtige Aspekte der Entwicklung des Strafvollzuges bzw. Jugendarrests in Gesetzgebung und Praxis nach der Föderalismusreform beleuchtet. Dies muss aber nicht das Ende der Debatte sein - den Autorinnen und Autoren in diesem Heft ist bewusst, dass man die Bilanz ausweiten könnte und sollte.

Prof. Dr. Heinz Cornel ist Professor für Jugendrecht, Strafrecht und Kriminologie an der Alice Salomon Hochschule Berlin und Mitherausgeber der Nenen Kriminalpolitik

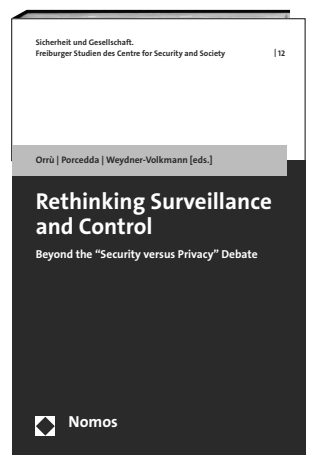

\section{Rethinking Surveillance and Control}

Beyond the „Security versus Privacy“ Debate

Herausgegeben von Dr. Elisa Orrú, Dr. Maria Grazia

Porcedda und Sebastian Weydner-Volkmann

2017, 240 S., brosch., 54,- $€$

ISBN 978-3-8487-3506-8

eISBN 978-3-8452-7809-4

(Sicherheit und Gesellschaft. Freiburger Studien

des Centre for Security and Society, Bd. 12)

nomos-shop.de/28291

Der Band versammelt Beiträge von namhaften Autoren aus Wissenschaft und Praxis zu aktuellen und relevanten Themen in der Sicherheitsdebatte. Er analysiert, welche Konsequenzen von Sicherheitsmaßnahmen für Bürger und Gesellschaft zu erwarten sind.

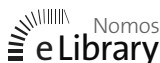

E Library Unser Wissenschaftsprogramm ist auch online verfügbar unter: www.nomos-elibrary.de

Bestellen Sie jetzt telefonisch unter (+49)7221/2104-37.

Portofreie Buch-Bestellungen unter www.nomos-shop.de

Alle Preise inkl. Mehrwertsteuer

Nomos 